\title{
Effect of uniform and non-uniform mesh on the development of turbulent boundary layer
}

\author{
Lokesh Kalyan Gutti*, Bhupendra Singh Chauhan** and Hee-Chang Lim*,*** \\ * School of Mechanical Engineering, Pusan National University, Busandaehak-ro 63beon-gil 2, Geumjeong-gu, Busan, Korea. \\ ** Department of Mechanical Engineering, GLA University, Mathura (UP), India. \\ *** Corresponding Author : hclim@pusan.ac.kr
}

\begin{abstract}
For incompressible flow simulation, it is commonly accepted to use uniform meshes to solve the governing equation of turbulent boundary layer. It follows the laws of conservation stabilizing the flow field in the domain and preventing odd-even decoupling in the pressure field. In this study, Large Eddy Simulation (LES) has been conducted in a long channel. In order to calculate the turbulent boundary layer in the channel, the unsteady Navier-Stokes equations has been adopted at a Reynolds number $=180$, which is based on mean centerline velocity and the half-width of the channel. The mesh used in this study was based on both stretch and uniform mesh having grid points, which is corresponding to . Turbulence statistics were also calculated to compare to the existing results. In the results, the turbulent boundary layer was fully developed at around . In addition, fully developed channel flow was achieved at the non-dimensional time of .
\end{abstract}

Keywords: Stretch grid; Large eddy simulation; Inflow generator, Turbulent boundary layer; Reynolds numbers.

\section{INTRODUCTION}

Fluid flow can be illustrated precisely by a set of partial differential equations. To achieve an exclusive result, the equations need to be added by initialing step and boundary conditions. On behalf of an extensive scope of momentum cases which is that shows an important challenge work in setting data point at the boundaries of the inlet. To generate turbulent inflow, condition the Reynolds stress tensor in the $\mathrm{c}$ language code (UDF) must be given according to the random fluctuation technique which should be multiplied with tensors and should be added with mean velocity. Many of the researchers are tried to collect new methods to generate realistic turbulent boundary layers using random fluctuation to solve complex problems. A review of some of them can be seen in Klein et al. (2003), Tabor et al. (1998), Xiaohua, (2017), Hung et al. (1996) \& Shaowen et al. (2014). At present, taking on both authenticated and new upgraded techniques are usually fulfilled within the framework of a specific CFD solver. Thus, various procedures can be able to access in various solvers utilizing a variety of programming languages and operations.

Tracking the direction of the wind (Wael et al., 2019; Navdeep et al., 2019; Abdelkader et al., 2019) on huge offshore wind turbine blades of around $10 \mathrm{MW}$ range, the profiles of wind and MPC yaw control are investigated in their research work.

The results are obtained from the c-code given at the inlet section in the initial stage. The long-standing aim of the code is to implement the realistic turbulent inflow generation technique. the execution of the code is done using technical programming. A set of advanced libraries for systematic processing is accessible to the users. All of these features create an outstanding platform to start a new inflow generation technique. 
The paper involves a large eddy simulation method (LES) to observe the boundary layer difference between uniform and stretch grids at different locations inside the channel. The reason to involve LES is more accurate than RANS simulation. The results of boundary layers are compared with DNS data (Myoungkyu et al., 2015) at some sections starting from inlet to pressure outlet.

In a wall-bounded turbulent shear flow, the power-law velocity profile in this paper is.

$$
u_{+}=C y_{+}^{\gamma}
$$

where $u_{+}=u / u_{+}$and $y_{+}=y u_{\tau} / v$. Further, $\mathrm{u}$ is the $\mathrm{x}$-direction velocity along the stream, $u_{\tau}=\sqrt{\tau_{w} / \rho}$ the friction of the skin, $\rho$ is the density of the fluid, and the molecular kinematic viscosity of a fluid is $v$. The power-law constants $\gamma$ and $\mathrm{C}$ are calculated as a function of the Reynolds number Re is fully formed turbulent channel flow.

\section{LES APPROACH}

The commonly used model (Nicoud et al., 1999) WALE (Wall-Adapting Local Eddy-viscosity) is used. The following form is available here.

$$
\mu_{t}=\rho \Delta_{s}^{2} \frac{\left(S_{i j}^{d} S_{i j}^{d}\right)^{3 / 2}}{\left(\bar{S}_{i j} \bar{S}_{i j}\right)^{5 / 2}+\left(S_{i j}^{d} S_{i j}^{d}\right)^{5 / 4}}
$$

Where the parameters are for the model is:

$$
\begin{gathered}
\mu_{t}=\rho \Delta_{s}^{2} \frac{\left(S_{i j}^{d} S_{i j}^{d}\right)^{3 / 2}}{\left(\bar{S}_{i j} \bar{S}_{i j}\right)^{5 / 2}+\left(S_{i j}^{d} S_{i j}^{d}\right)^{5 / 4}} \\
\Delta_{s}=C w(\Delta x \Delta y \Delta z)^{1 / 3} \\
S_{i j}^{d}=\frac{1}{2}\left(\bar{g}_{i j}^{2}+\bar{g}_{j i}^{2}\right)-\frac{1}{3} \delta_{i j} \bar{g}_{k k}^{2} \\
\bar{g}_{i j}=\frac{\partial \bar{u}_{i}}{\partial x_{j}}
\end{gathered}
$$


The operation of Newtonian fluids of constant density at constant temperature is governed by the equations of incompressible Navier Stokes (Shaowen et al., 2014).

$$
\frac{\partial u_{i}}{\partial t}+\frac{\partial}{\partial x_{j}}\left(u_{i} u_{j}\right)=-\frac{1}{\rho} \frac{\partial p}{\partial x_{i}}+v \frac{\partial^{2} u_{i}}{\partial x_{j} \partial x_{j}}, \quad \frac{\partial u_{i}}{\partial x_{i}}=0
$$

Here, represents the -component of the flow velocity field and the pressure is shown by. The viscosity of the density and the kinematic viscosity is represented by the respective and. For repetitive indices, Einstein's summation convention is assumed. It is not possible to calculate the most functional turbulent flows directly from the Navier-Stokes equations, Equation 6, that there is usually an inadequate resolution to address all applicable scales of motion. Hence, a large eddy simulation is chosen to estimate the large length scale conduct of turbulent flows. In the large-eddy simulation, the difference between small and large-scale motion is generally extracted by a filtering or coarse-graining operation. Filtered incompressible Navier-Stokes equations can properly describe the evolution of large-scale incompressible velocity fields (Shaowen et al., 2014).

$$
\frac{\partial \bar{u}_{i}}{\partial t}+\frac{\partial}{\partial x_{j}}\left(\bar{u}_{i} \bar{u}_{j}\right)=-\frac{1}{\rho} \frac{\partial \bar{p}}{\partial x_{i}}+v \frac{\partial^{2} \bar{u}_{i}}{\partial x_{j} \partial x_{j}}-\frac{\partial}{\partial x_{j}} \tau_{i j}, \quad \frac{\partial \bar{u}_{i}}{\partial x_{i}}=0
$$

The interactions between large and small scales of motion are represented by turbulent, or subfilter-scale, stresses, $\tau_{i j}=\bar{u}_{\imath} u_{\jmath}-\bar{u}_{i} \bar{u}_{j}$. Since it is not explained entirely in terms of the large-scale field of velocity, they must be modeled. Turbulent models for stresses in the large-eddy simulation $\tau_{i j}^{\bmod }(v)$, such that there is a differential equation given by

$$
\frac{\partial v_{i}}{\partial t}+\frac{\partial}{\partial x_{j}}\left(v_{i} v_{j}\right)=-\frac{1}{\rho} \frac{\partial q}{\partial x_{i}}+v \frac{\partial^{2} v_{i}}{\partial x_{j} \partial x_{j}}-\frac{\partial}{\partial x_{j}} \tau_{i j}^{\bmod }(v), \frac{\partial v_{i}}{\partial x_{i}}=0
$$

The filtered velocity $v_{i} \approx \bar{u}_{i}$ and pressure $q \approx \bar{p}$, provide precise approximations. We will be pointing to Equation 8 , as the large-eddy simulation (basic) equations. In these equations, we have intentionally lowered the overbars because without clear filtering we can concentrate on large-eddy simulation which od discussed by Oberlack, (1997) and Dina et al. (2007). The equations for large-eddy simulation are presented in Equation 8, are numerically resolved. In the current work, this step which includes discretization and is closely linked to the modeling process is not explored in depth.

\section{COUPLING ALGORITHM}

In a segregated or combined way, the PBCS used to solve flow models there are so many benefits using coupled scheme through depends on which flow model we choose. For steady-state flows, the coupled system achieves a stable and reliable single-phase implementation, first, it starts with initializing the beginning loop to solve the case, next based on our model type we can either choose a segregated pressure-based solver, pressure-based coupled solver (PBSC) or density-based coupled solver (DBCS). In my research work, I choose a pressure-based coupled solver (PBSC) which solves both combination of $U, v$ and $w$ momentum and mass continuity equations together. There is an advantage by solving in this way is to overcome the divergence problems and makes the simulation accurate and smooth way. 
After completed solving the mass and momentum equation it directly jumps through the energy equation which comes from three velocities $U, v$ and $w$ through the initialization process. Then enters to the species to solve turbulence equations which are very important for turbulence models that decide the Reynolds stress flows. At every given iteration, the coupled scheme always tries to overcome the pressure corrections through transport equations as required.

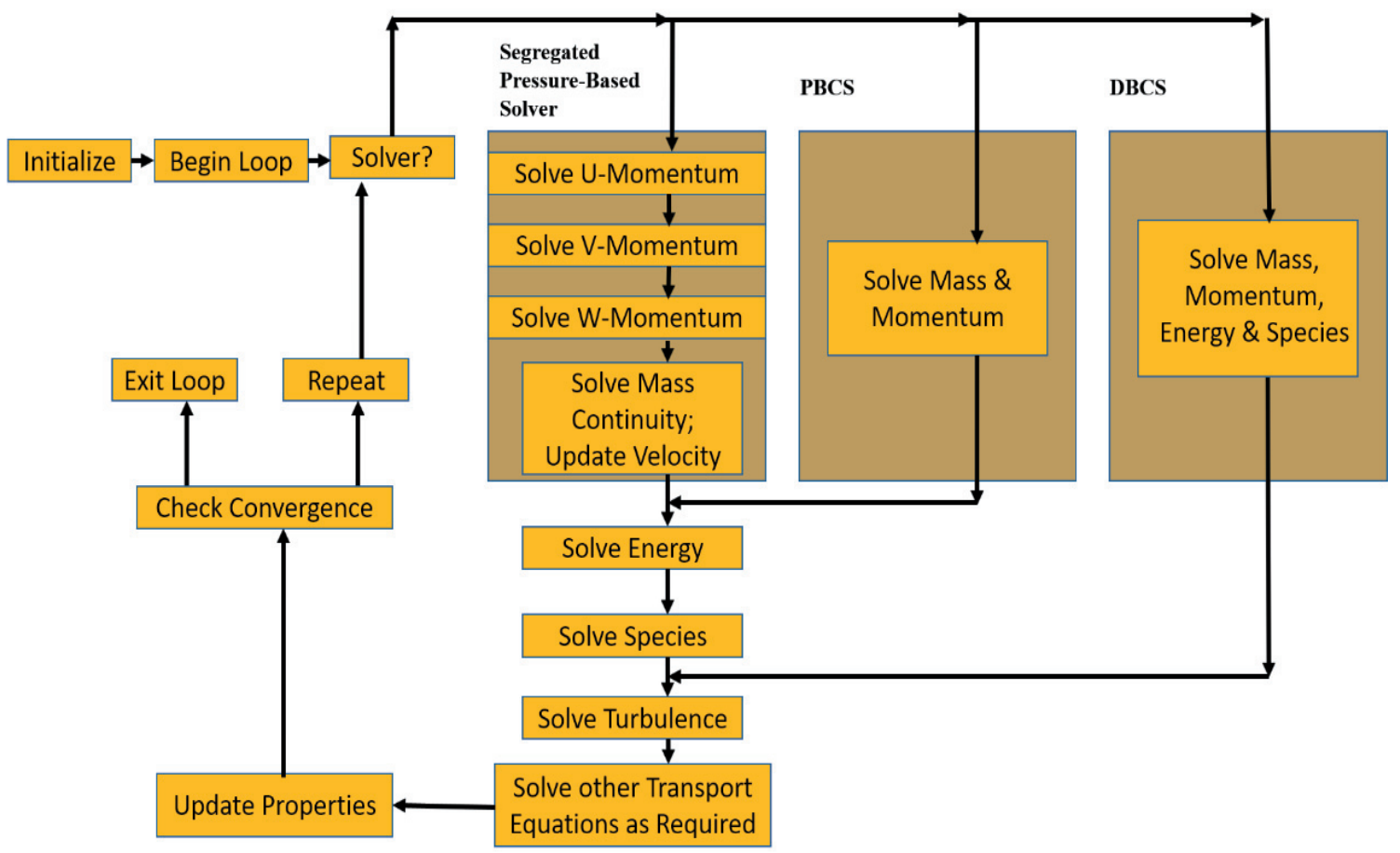

Figure 1. Sketch of the coupling scheme solver

After reaching some timesteps transport equation addresses all three velocity $U, v$ and $w$ properties to update to check the convergence point are reached or not. If not, the process exits the loop and shoed the divergence mostly in momentum equation are not proper match to given initial velocities. If the solution is converged, then it repeats the process until that reaches the end of the time steps. But most of the cases coupling scheme will not prefer to get pressure corrections in between the iterations. The density-based coupling scheme will also be processed in the same way, but it directly solves from species which are updated previous steps. This density model's maximum implicit coupling is achieved.

\section{DETAILS OF A DOMAIN MODEL}

The computation domain size for both uniform and stretch the channel flow was $6.36 \pi d \times 2 d \times 2 d$ as shown in Figure 2. Where $d$ represents the half depth of the channel. In this section, the axis system is the common 'engineering' system with $y$-coordinate normal to the wall. As (John et al., 1986) the Reynolds number was 180 based on the friction velocity and the half depth of channel $d$. A mesh of $200 \times 96 \times 96$ was used; it was extended from the walls in the $y$-direction to the total height with a 1.3 grid space wall unit in the $y$-direction and a uniform grid in another domain. 


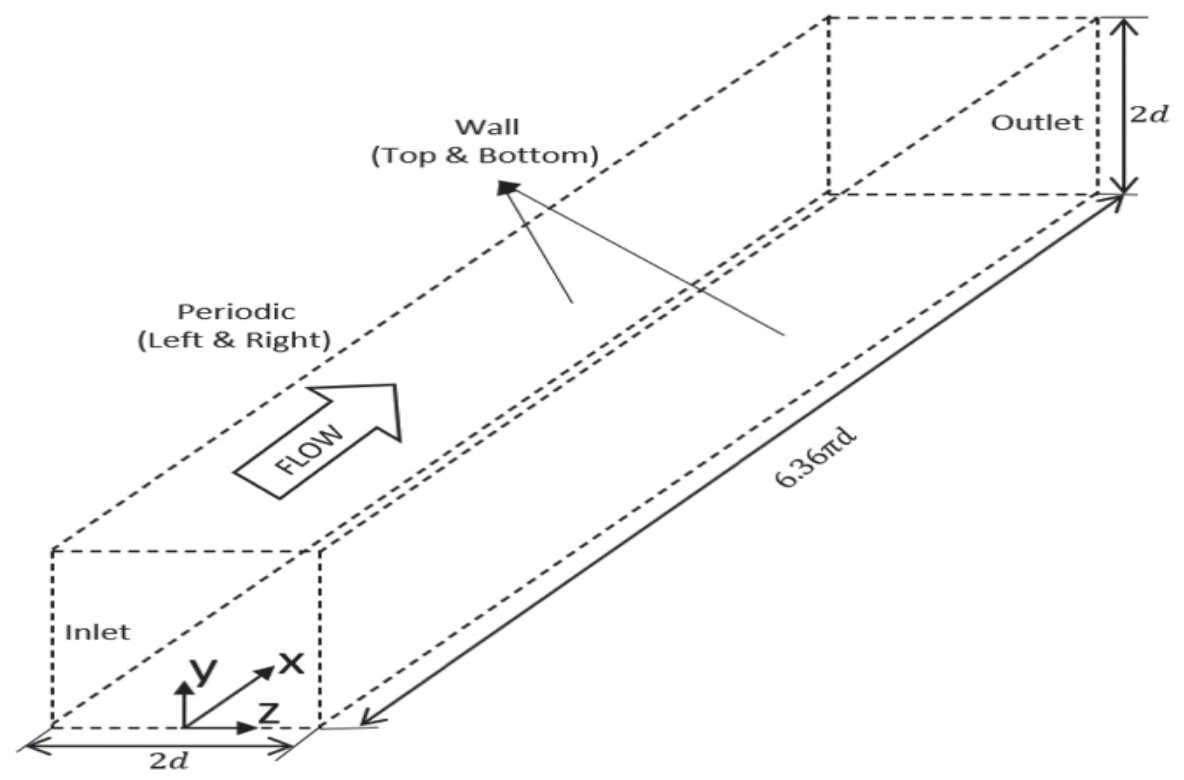

Figure 2. Schematic view of the domain model

Table 1. Summary of the simulation parameters

\begin{tabular}{|c|c|}
\hline Name & Size \\
\hline & 200 \\
\hline Grid spanwise nz & 96 \\
\hline Kinematic viscosity (nu) & 96 \\
\hline Channel width (d) & 0.5 \\
\hline Average streamwise velocity (Umean) & 1 \\
\hline & \\
\hline First cell heigh from the wall & \\
\hline & \\
\hline (N) & \\
\hline
\end{tabular}




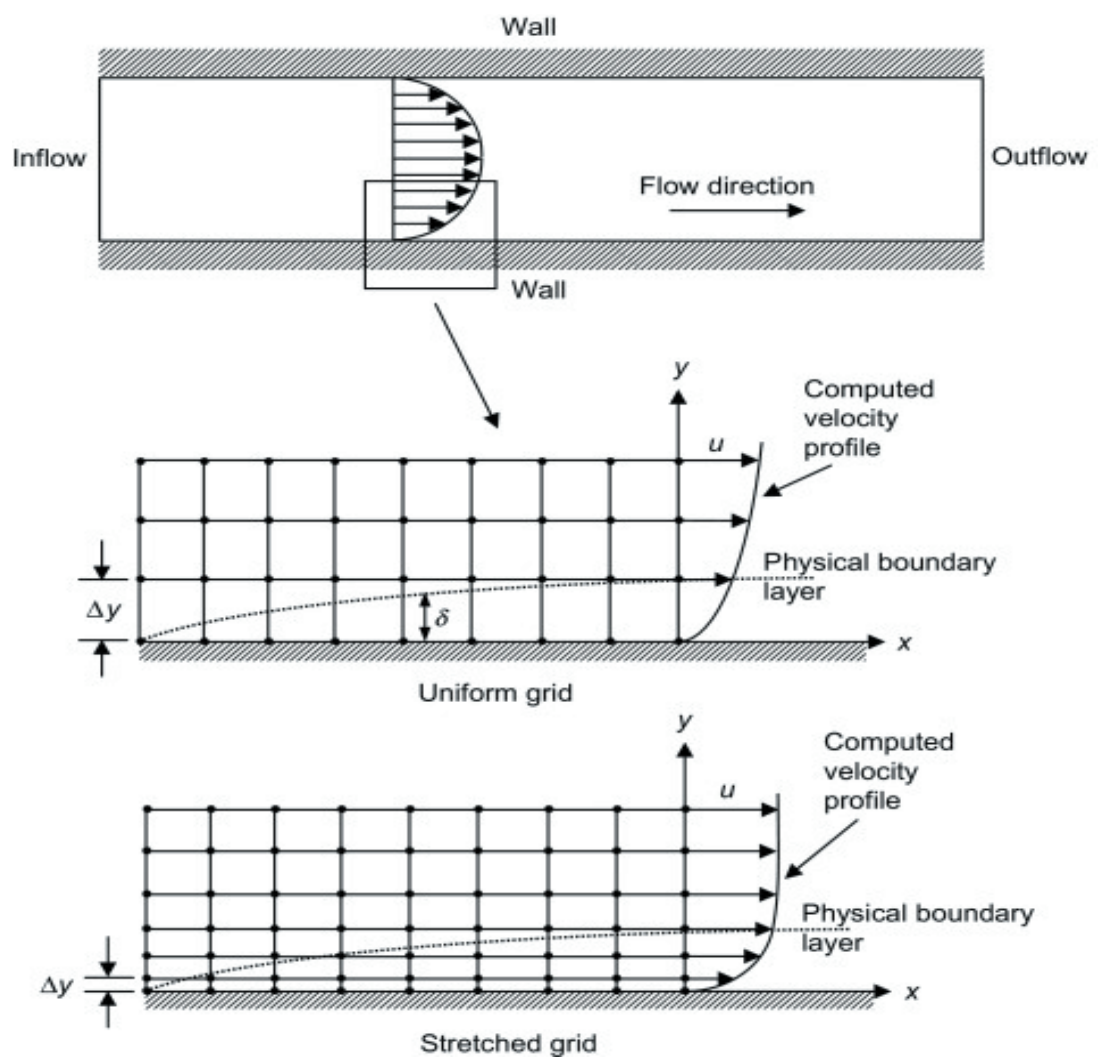

Figure 3. Schematic view of uniform and stretch mesh

\section{MESHING}

Figure 4 and 5 shows Mesh is another crucial stage of a CFD simulation, which should be consistent with the precise simulation of the atmospheric boundary layer as well as fluid flow near the wall. Therefore, some of the meshing factors for instance from the wall, first node height, to conduct efficient simulations, the number of nodes up to the height should be kept within certain limits. A mesh provides precise outcomes for both laminar flow and turbulent flow conditions. It is possible to subdivide the atmospheric boundary layer into some regions. That must be solved based on the region, the location of the first cell adjacent to the wall is defined in Table 1. Enhanced wall treatment should be used when the flow characteristic in the viscous sub-layer needs to be captured. When the flow resolution begins from the log-layer area, standard wall functions can be employed. Some limitations on the placement of the first cell from the wall are imposed depending on the choice of near-wall treatment but uniform mesh needs to take care of CFL number at every time step.

\section{DETAILS OF NUMERICAL SETUP}

The present LES WALE model is implemented in a general CFD code, ANSYS Fluent 19.1 version, depending on the approach of finite-volume adopted for the present numerical simulations. The modeling of LES-WALE turbulence is implemented via UDFs in the code (User Defined Function). Using a second-order upwind system, the convective terms are discretized. For the turbulence model equations, the second-order upwind method is used, and for temporal discretization, a second-order implicit scheme is used. In pressure-velocity coupling, the Semi Implicit Approach for 
Pressure-Linked equations (Coupled) algorithm is used. The time step is $2.5 \times 10^{-4} \mathrm{~s}$, which in most regions results in a CFL number smaller than 0.95 . the simulations are averaged for more than $1 \times 10^{5}$ time steps.

\section{PLANE CHANNEL FLOW}

Then, we concentrate on a turbulent plane-channel flow with large-eddy simulations. For this case study, the reference data comes from the Direct Numerical Simulation (DNS). The mean velocity $u_{i}$, and the Reynolds stresses, which are averaged over time were collected among other statistical quantities. Using the WALE model Equation 2, the large-eddy simulations were carried out. To match reference data, Reynolds number based on the half depth of the channel $R e_{\tau} \approx 180$ (John et al., 1986). For both uniform and stretch grids, the domain size is $6.36 \pi d \times 2 d \times 2 d$ Where represents the half depth of the channel. Uniform mesh for streamwise $\left(x_{1}\right)$ and spanwise $\left(x_{3}\right)$ directions, $\left(x_{2}\right)$ whereas indicates mesh is extended (stretched) in a normal direction.

The profiles of mean velocity and the Reynolds stresses using the WALE model of the large-eddy simulations are shown in Figures 6 and 7. For comparison of results reference DNS data from Myoungkyu et al. (2015) is shown. To make a comparison between the direct numerical simulation stresses of Reynolds and large-eddy simulations using eddy viscosity methods which is the WALE model, we show only the normal stresses, Reynolds stress and mean velocity profiles at some sections in a plane channel flow. Both results are normalized with dependents on the velocity of friction $u_{\tau}$, and the half-width of the channel $d$.
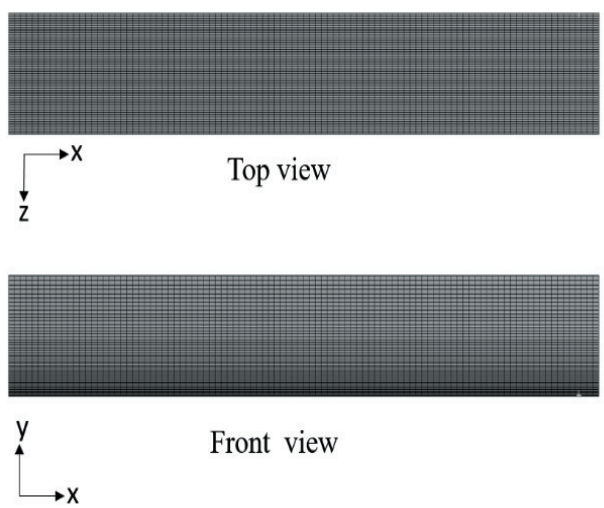

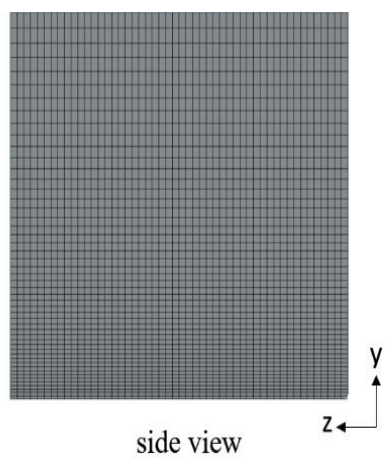

side view

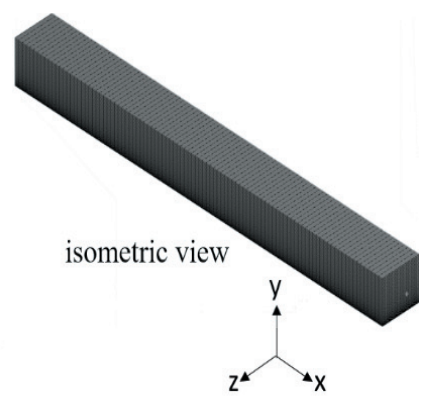

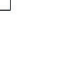

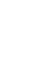

Figure 4. Domain with mesh label stretch
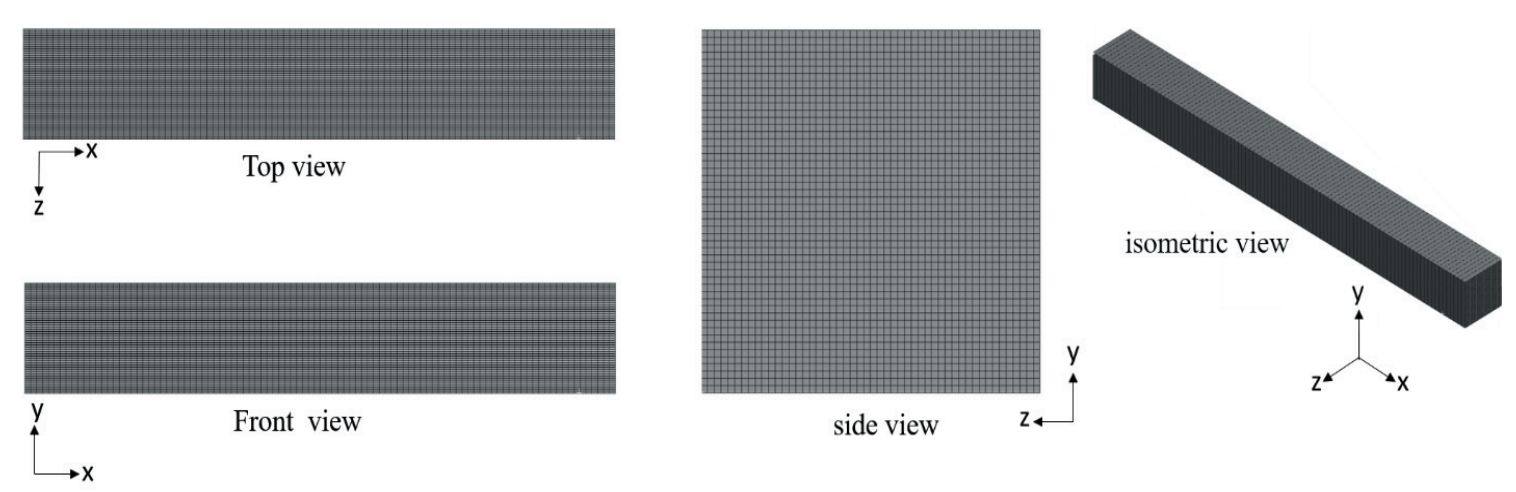

Figure 5. Domain with mesh label uniform 


\section{RESULTS AND DISCUSSION}

\subsection{Vertical Profiles of Mean Flow}

In this study, the formation of a turbulent boundary layer at the inlet flow is the challenging work to get a match with the DNS data (Myoungkyu et al., 2015) from upstream to downstream $(x / d=$ inlet, 04,06,08,10,12,14,16,18 and 20) for both cases of stretch and uniform mesh in Figure 6 using the LES WALE model.

Figure 6 shows a stretch grid of mean velocity boundary layer profiles that have similar behavior with DNS data (Myoungkyu et al., 2015) from the height of $y / d=0.5$ to $y / d=2$. But profiles near the wall have some minor differences when comparing with DNS results. This is due to the much less accurate wall functions in this region and the turbulence models used within the laminar sublayer may not be able to account for the effects of the low-Reynoldsnumber based on friction velocity which is $R e_{\tau}=180$.

A uniform grid of mean velocity profiles that has the same kind of trend follows a stretch grid. This is due to the same kind of inlet condition given to the uniform case also, however as we expect this kind of behavior from a uniform case that has some effect near the wall while comparing with DNS data (Myoungkyu et al., 2015).

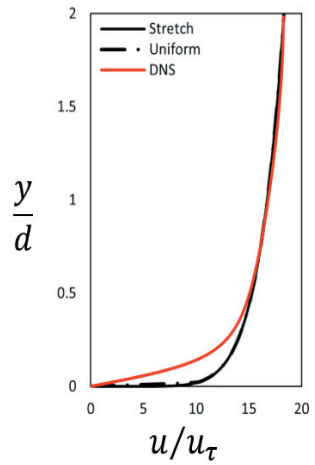

$x / d$

Inlet

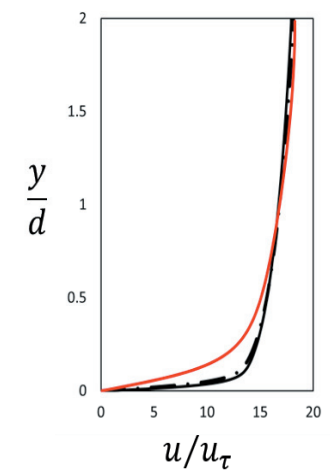

$x / d$

12

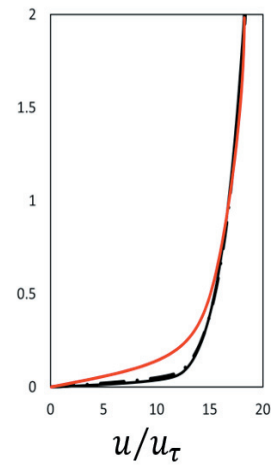

04

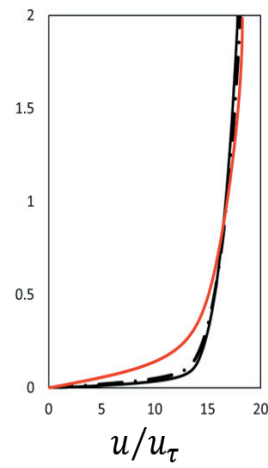

14

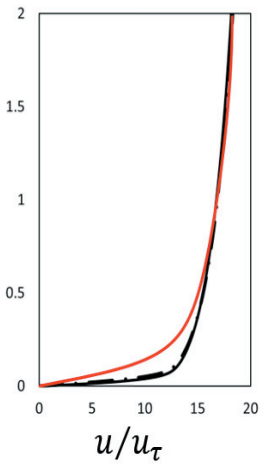

06

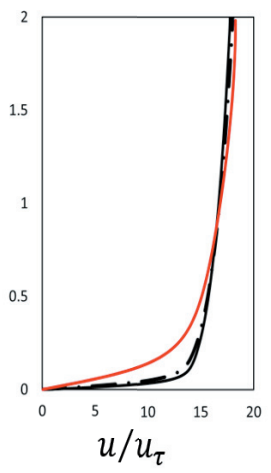

16

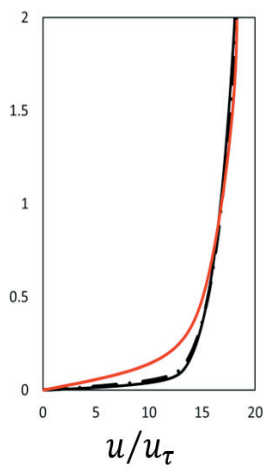

08

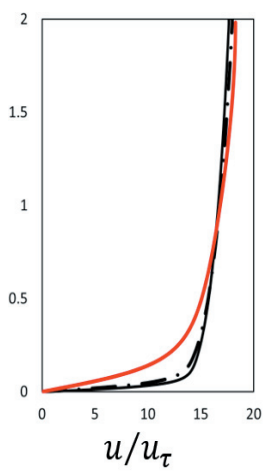

18

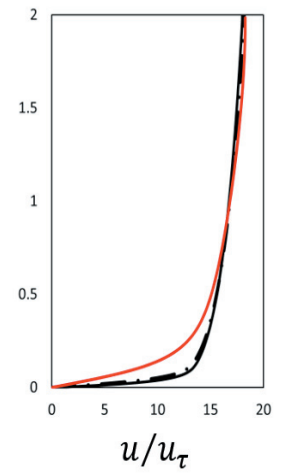

10

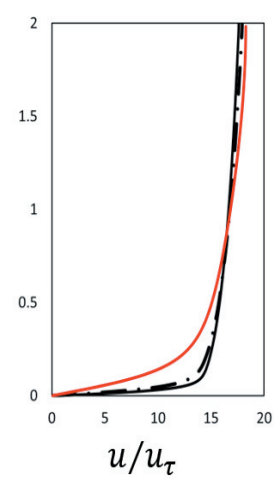

20

Figure 6. Comparison of boundary layer profiles with DNS data (Myoungkyu et al., 2015) at each streamwise locations for stretch and uniform mesh 


\subsection{Fluctuation Components of Axial and Reynolds Stress Profiles}

Figure 7 shows the fluctuation components of normal and Reynolds stress for both cases of stretch and uniform compared with DNS data (Myoungkyu et al., 2015) which is normalized with friction velocity $u_{\text {tau }}$ and measured at downstream $x / d=10$ of the computational domain.

In the case of stretch the magnitude of fluctuating component $u$ ' $u$ ' from the wall is close to the DNS result and from the wall to some height, very minor differences can be observed. In the end, it gives the same trend and almost close magnitude with DNS. In the case of uniform, it seems difficult to match either with stretch or DNS near the wall or far away from the wall. But the end of the height from $y / d=1.5$ to $y / d=2$ the fluctuating component $u$ ' $u$ ' follows both DNS results as well as a stretch case.

(a)

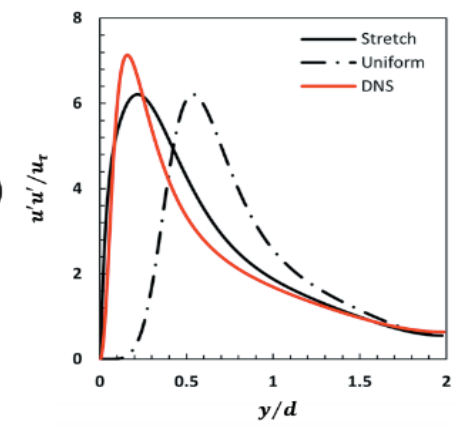

(c)

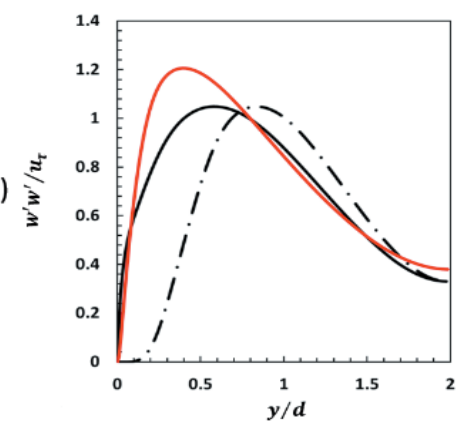

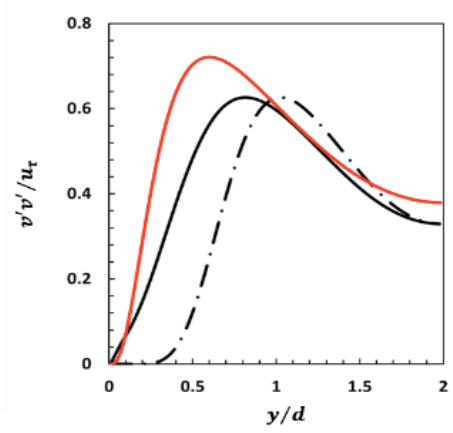

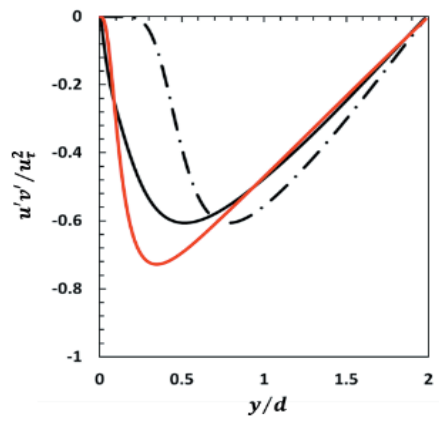

(b)

(d)

Figure 7. Comparison of normal stress and Reynolds stress profiles with DNS data (Myoungkyu et al., 2015) at streamwise location $\mathrm{x} / \mathrm{d}=10$ for stretch and uniform mesh

The normal stress components of $v^{\prime} v^{\prime}$ and $w^{\prime} w^{\prime}$ when it got compared with DNS data (Myoungkyu et al., 2015). Stretch mesh case has almost close values up to the height $y / d=0.25$ which is near the wall but in the case of uniform mesh the magnitude of the fluctuating components near the wall is almost zero up to $y / d=0.25$ from there it follows the same trend with DNS data (Myoungkyu et al., 2015) and stretch mesh case as well.

Finally, the Reynolds stress component $u$ ' $v$ ' which is normalized with friction velocity $u_{t}$, the flow trend between two cases stretch grid and uniform grid having a difference in magnitude while height increases. But in the case of a stretch from the height $y / d=0.5$ that has a close match with DNS data (Myoungkyu et al., 2015). For uniform, some difference can be observed from the same height in terms of the magnitude of $u$ 'v' but it also follows the universal flow field from the mid-height on words. 


\section{CONCLUSION}

The usage of the coupling algorithm is the best suitable way for steady and unsteady state problems to avoid pressure correction problems at the outlet and divergence of $x, y$ and $z$ momentums in between the simulation process.

The LES WALE model is one of the selected models for simulating the atmospheric turbulent boundary layer flow. The turbulent boundary layer is well developed to compare with DNS data (Myoungkyu et al., 2015). However, only the drawback is the near-wall region at every location up to $x / d=20$ for both stretch and uniform case.

The normal stress and Reynolds stress for stretch mesh case is almost well-formed but in the case of uniform it becomes hard to develop near the wall which is almost zero values up to the height of $y / d=0.25$.

In this study, there are some minor things to implement in our work in the future. Firstly, the main drawback in terms of turbulent boundary results for both stretch and uniform case not close to DNS data (Myoungkyu et al., 2015) at near the wall only. Maybe it is due to velocity near the wall region is faster growing in case of both stretch and uniform results. We need to increase the length of the computational domain to make it move more closer to DNS results.

In addition, further research is needed in the case of ramp obstacle channel flow to observe flow separation visuals by obstacle and vortex shedding structures at the downstream locations. Deficit velocity wake locations are an important feature to identify the added momentum which turbulent results of this study are used at inlet plane of ramp model domain case.

\section{ACKNOWLEDGMENT}

This work was supported by 'Human Resources Program in Energy Technology' of the Korea Institute of Energy Technology Evaluation and Planning (KETEP), granted financial resource from the Ministry of Trade, Industry \& Energy, Republic of Korea (no. 20184030202200). In addition, this work was supported by the National Research Foundation of Korea (NRF) grant funded by the Korea government (MSIP) (no. 2019R1I1A3A01058576). This work was also supported by the National Supercomputing Center with supercomputing resources including technical support (KSC-2020-INO-0025).

\section{REFERENCES}

John, K., Parviz, M. \& Robert, M. 1986. Turbulence statistics in fully developed channel flow at low Reynolds number. J. Fluid Mech, 177: 133-166.

Tabor, G. \& Baba, M.H. 1998. On the generation of turbulent inflow conditions for boundary layer simulations. J. Comput Phys, 140: 233-58.

Xiaohua, W. 2017. Inflow turbulence generation methods. Annu Rev Fluid Mech, 49:23-49.

Hung, L., Parviz, M. \& John, K. 1996. Direct numerical simulation of turbulent flow over a backward-facing step. J. Fluid Mech, 349-374.

Shaowen, F., Xingfei, Y., Ruojun, Q. \& Shuhui, J. 2014. Finite Element Solution in Fluid Mechanics using exponential function based interpolation. Applied Mechanics and Materials, 3051-3056.

Nicoud, F. \& Ducros, F. 1999. Subgrid-scale stress modelling based on the square of the velocity gradient tensor. Flow, Turbulence and Combustion, 62: 183-200. 
Klein, M., Sadiki, J. \& Janicka, J. 2003. A digital filter based generation of inflow data for spatially developing direct numerical or large eddy simulations. J. Comput Phys, 186: 652-665.

Oberlack, M. 1997. Invariant modeling in large-eddy simulation of turbulence. Center for Turbulence Research, pp. $3-22$.

Dina, R., Aziz, H. \& Martin, O. 2007. Analysis and development of subgrid turbulence models preserving the symmetry properties of the Navier Stokes equations. Eur. J. Mechanics B/Fluids, 26: 531-550.

Robert, D.M., John, K. \& Nagi, N.M. 1999. Direct numerical simulation of turbulent channel flow up to $\operatorname{Re} \tau=590$. J. Phys. Fluids, 11: 943-945.

Myoungkyu, L. \& Robert, D.M. 2015. Direct numerical simulation of turbulent channel flow up to $\operatorname{Re}$ tau $=5200$. J. Fluid Mech, pp. 395-415.

Lund, T. S., Wu, X. \& Squires, K. D. 1998. Generation of turbulent inflow data for spatially-developing boundary layer simulations. J. Comput Phys, 140: 233-258.

Wael, F., Manal, E.H., Ahmed, K. \& Khaled, E.M. 2019. Analysis of various yaw control techniques for large wind turbines. Journal of Engg. Research, pp. 215-231.

Navdeep, S., Bhanu, P. \& Akhilesh, S. 2019. Nonlinear robust observer based adaptive control design for variable speed wind turbine. Journal of Engg. Research, pp. 258-285.

Abdelkader, L., Fateh, k. \& Abdelhalim, B. 2019. Model predictive control for an SAPF interfaced by a wind energy conversion system based on permanent magnet synchronous generator. Journal of Engg. Research, pp. 1-19. 